\title{
Assessment of Patient and Provider Satisfaction With the Change to Telehealth From In-Person Visits at an Academic Safety Net Institution During the COVID-19 Pandemic
}

\author{
Judith Volcy, DO, Walkitria Smith, MD, Krystal Mills, MD, Ashley Peterson, DO, \\ Ijeoma Kene-Ewulu, MD, Macy McNair, MD, Riba Kelsey, MD, and Nkechi Mbaezue, MD
}

Introduction: The 2019 novel coronavirus (COVID-19) caused a global pandemic that forced medical providers to rapidly alter methods of health care delivery. One month into this pandemic, we surveyed providers and patients to assess satisfaction or concerns with the change from in-person visits.

Methods: We surveyed internal medicine (IM) and family medicine (FM) faculty and residents to ascertain satisfaction or concerns with the change to telehealth from in-person visits.

Results: Of patients surveyed (129 IM, 94 FM), 84.4\% of IM patients and 94\% of FM patients agreed or strongly agreed that they enjoyed the televisits, and $82.9 \%$ of IM providers ( 47 surveyed) and $64 \%$ of FM providers (25 surveyed) felt the same. For continued televisits, $76.74 \%$ of IM patients and $84.1 \%$ of FM patients agreed or strongly agreed that they would not mind having virtual visits after the pandemic, compared with $89.44 \%$ of IM providers and $88 \%$ of FM providers, and $91 \%$ of IM providers and 88\% of FM providers felt comfortable managing visits virtually.

Conclusion: Patients are open to the expanded use of telemedicine, and providers and hospital systems should be prepared to embrace it for the benefit of patient care. ( $\mathrm{J}$ Am Board Fam Med 2021;34:S71-S76.)

Keywords: COVID-19, Delivery of Health Care, Pandemics, Patient Care, Personal Satisfaction, Surveys and Questionnaires, Telemedicine

\section{Introduction}

The COVID-19 pandemic forced physicians to abandon the traditional visit, where patients and physicians meet face-to-face, and shift to a socially distant visit, where providers call patients for either a phone visit or a video visit to conduct medical care. In the United States, the percentage of providers using audio or video technology for office visits was $11 \%$ pre-COVID,${ }^{1} 69 \%$ during the height of COVID in April 2020, and 21\% of total

This article was externally peer reviewed.

Submitted 26 July 2020; revised 6 August 2020; accepted 16 November 2020.

From the Department of Medicine, Morehouse School of Medicine, Atlanta, GA (JV, KM); Department of Family Medicine, Morehouse School of Medicine, Atlanta, GA (WS, AP, MM, RK, NM); and VA Southeast Network (VISN 7) Tele Primary Care, Duluth, GA (IKE).

\section{Funding: None.}

Conflict of interest: None.

Corresponding author: Judith Volcy, DO, Department of Medicine, Morehouse School of Medicine, 720 Westview Drive, SW, Atlanta, GA 30310 (E-mail: jvolcy@msm.edu). encounters in mid-July 2020. ${ }^{2}$ Virtual visits were a necessity that allowed for patients to continue to receive care for their chronic and nonemergent acute conditions. As providers at an academic institution caring for the underserved, we were interested in finding out how our patients, faculty, and learners were feeling during this whirlwind of change from traditional visits to virtual. We surveyed patients and providers during the height of the pandemic to ascertain satisfaction or concerns with the change to telehealth from in-person visits.

\section{Methods}

After securing Institutional Review Board approval, we surveyed residents and faculty at 2 practice locations during the height of the COVID-19 pandemic to see how they felt conducting office visits via audiovisual technology. The internal medicine (IM) residents and faculty saw patients in the hospital clinic. Family medicine (FM) residents and faculty saw 
patients in a freestanding community clinic. IM faculty and residents were asked to survey patients between April 16 and April 30, 2020 on their telemedicine experience, which was done entirely over the phone. FM patient surveys were collected from April 22 through May 15, 2020, with the majority of FM visits by video (approximately 80\%) and the remaining by phone. The video visits were done on the platform Procle.

The residents, who spanned all 3 years of training, were those rotating through the clinic as well as those faculty assigned to the precept clinic or there to see their own patients. Before each clinic session, the providers were reminded about the survey and given a copy of the questions. For ease of accessing the questions, the questions were made available to the providers by e-mail and by hard copy. The providers were tasked with asking the patients at the end of the visit if they would be interested in participating in a short survey about how they felt about the audiovisual visit. If they agreed, the questions would then be included in the survey.

The 3 questions were as follows:

1. I enjoyed having my visit done via virtual visit/ telemedicine.

2. I wouldn't mind having virtual visits/telemedicine visits at least part of the time, even after the public health emergency.

The answer choices for the first two questions were on a Likert scale: A: Strongly agree, B: Agree, C: Undecided, D: Disagree, or E: Strongly Disagree.

The third question was a self-assessment of health:

3. I rate my own overall health as the following:

The answer choices for the third question were A: Excellent Health, B: Good, C: Fair, D: Poor, or E: Very Poor.

The surveys were anonymous, with the providers recording patient age, sex, and race. As all patients were not on site, the questions were read to the patients, and their responses were then recorded by the providers. The provider surveys were done at the end of the clinic sessions. The IM providers were able to either fill out a hard copy of the survey, text their responses back, fill out a questionnaire on SurveyMonkey, or verbally give their responses to be recorded. Residents were asked to fill out 1 survey. The FM providers used SurveyMonkey exclusively to record their answers.

The providers used the same Likert scale as above. The survey questions were as follows:
1. I enjoyed seeing patients via this modality.

2. I felt comfortable managing patients via this modality.

3. If I had a choice, I wouldn't mind seeing my patients in this way, at least a portion of the time.

They then had to identify their year of training, residency (IM or FM), or indicate that they were faculty. The provider surveys were anonymous except for those recorded using SurveyMonkey.

\section{Results}

Two hundred thirty virtual visits were conducted between April 16 and April 30, 2020 for IM with a $75 \%$ show rate. One hundred twenty-nine patients $(56 \%)$ agreed to participate in the survey. Four hundred seven patients were seen by FM providers between April 30 and May 15, 2020. Ninety-four patients $(23 \%)$ agreed to participate in the survey. Some of the patients declined virtual visits after being informed that there might be a charge for the virtual visit, albeit a fraction of what the in-person visit would cost. Others preferred to be rescheduled when in-person visits became available again.

Figure 1 summarizes the patient responses to the survey. Nearly all (84.4\% of IM patients and $94 \%$ of FM patients) patients agreed or strongly agreed that they enjoyed the telemedicine visits. A smaller percentage of patients $(76.74 \%$ of IM respondents vs $84.1 \%$ of FM respondents) agreed or strongly agreed that they would not mind having virtual or telemedicine visits at least part of the time, even after the public health emergency. Figure 2 and Figure 3 summarize patient responses to how they assess their own health and matches those self-assessments with their answers to their feelings about virtual visits. The largest percentage of respondents in both groups rated their health as good across all categories. For IM, that number was $73.6 \%$ of respondents, and for FM, the number was $58.5 \%$. The fewest number of IM clinic patients considered their health to be very poor $(0.78 \%)$, but the next lowest group was actually the group who considered themselves to be in fair health, at $6.2 \%$.

For IM respondents, $13.17 \%$ considered themselves to be in poor health. The worse the patients assessed their health, the less likely they were to rate their virtual visits as positive, and the less likely they were to be open to future virtual visits, although the differences were not statistically 
Figure 1. Patient survey responses.

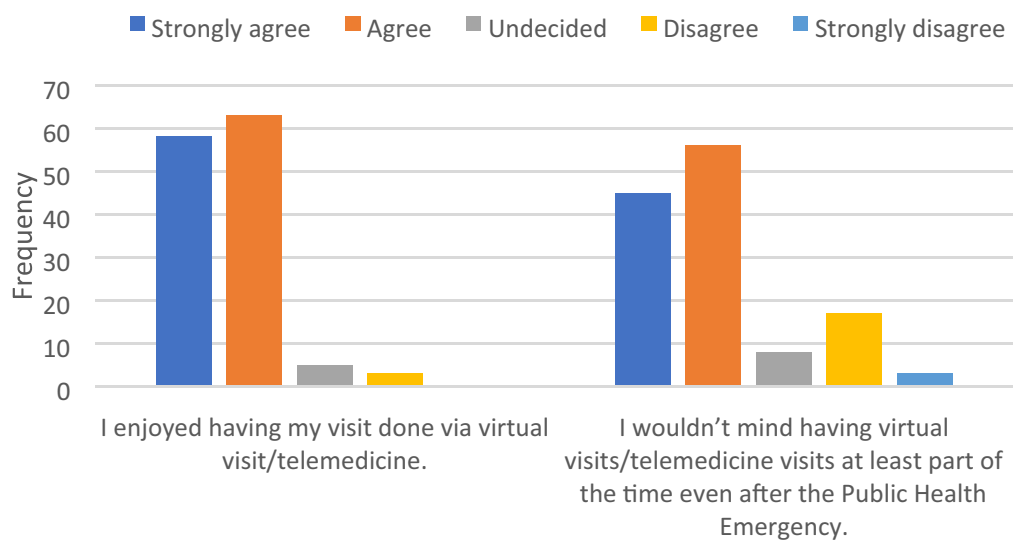

significant. Age-related difference in visit enjoyment and receptiveness to future visits was statistically significant for the FM patients $(P<0.000)$. However, when controlled for gender and health rating, statistical significance was not maintained. There was also no statistically significant difference between the impact of either gender or self-assessed health rating on virtual visit enjoyment or receptiveness to future virtual visits.

Only $11.6 \%$ of IM patients considered themselves to be in excellent health. For FM respondents, a slightly higher number, $14.9 \%$, considered themselves to be in excellent health; $21.3 \%$ reported to be in fair health and $5.3 \%$ in poor health. None reported feeling that they are in very poor health.

The IM arm of the study failed to show statistical significance in any of the questions.
There was no statistical significance between the different age groups who reported enjoying the visit $(P=.1839)$ or in those who replied that they would not mind having some future visits via telemedicine $(P=.4462)$. There was also no statistical difference found between the groups as they responded to how they would describe their health $(P=.4397)$.

Surveys were collected from 47 IM providers. Eight (17\%) were faculty and 39 (89\%) were residents across all 3 postgraduate year levels. This represents a response rate of $61.5 \%$ of faculty and $72 \%$ of rotating residents. Surveys were collected from 25 FM providers consisting of 7 faculty (28\% of respondents) and 18 residents ( $72 \%$ of respondents) across all 3 postgraduate year levels. This represents a $41 \%$ faculty response rate and a $100 \%$ resident response rate. Figure 4, Figure 5, and Figure 6 summarize the provider responses to the survey

Figure 2. Patient survey responses regarding satisfaction with their virtual visit compared with self-assessment of health status.

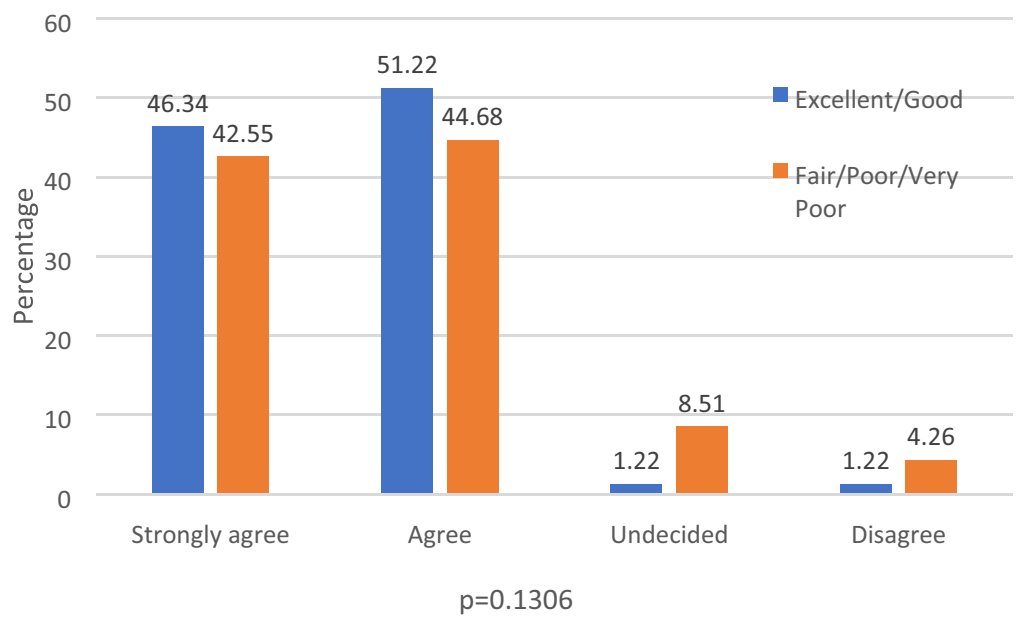


Figure 3. Patient survey responses as to whether they would be open to continued virtual visits after the pandemic. Responses were crossed with patient assessment of health.

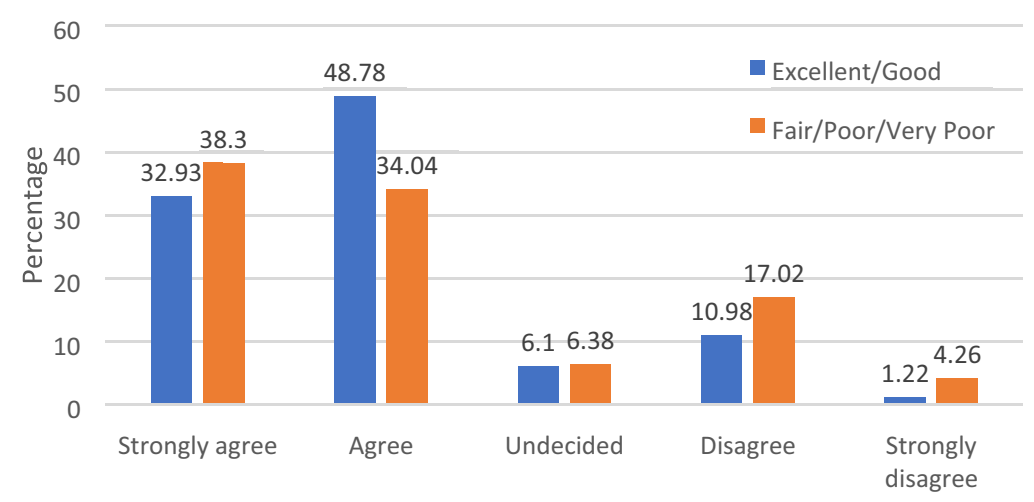

$\mathrm{p}=0.4397$

questions per level of training. Only less than $20 \%$ of postgraduate year 3s answered strongly agree to question 1 , whereas $50 \%$ of faculty answered strongly agree. The Fisher's exact test showed no statistically significant differences. No IM providers strongly disagreed that they enjoyed seeing patients virtually, compared with $4 \%$ of FM providers who disagreed. For IM providers, $82.89 \%$ strongly agreed or agreed that they enjoyed seeing patients virtually; 64\% of the FM providers strongly agreed or agreed that they enjoyed the virtual visits. For feeling comfortable managing patients virtually, $91.4 \%$ of IM providers strongly agreed or agreed that they felt comfortable doing so; $88 \%$ of FM providers strongly agreed or agreed that they were comfortable with conducting these visits. As to the future,
$89.44 \%$ of IM providers and $88 \%$ of FM providers strongly agreed or agreed that they would see patients virtually, if given the opportunity.

There was no statistical difference seen among IM providers who responded that they enjoyed seeing patients via telemedicine $(P=.4286)$ or in the groups who felt comfortable seeing patients via telemedicine $(P=.5957)$, nor in those who replied that they would not mind seeing future patients at least part of the time via telemedicine in the future $(P=.4493)$. The majority $(77.5 \%$ for IM and $92.5 \%$ for FM) of patients were African American. Considerably more women than men completed the survey, with $77.5 \%$ of the IM patients and $79.8 \%$ of the FM patients self-identifying as female. The average age of the patients was 57.7 years.

Figure 4. Provider response to the statement "I enjoyed seeing patients via this modality." Abbreviation: PGY, postgraduate year.

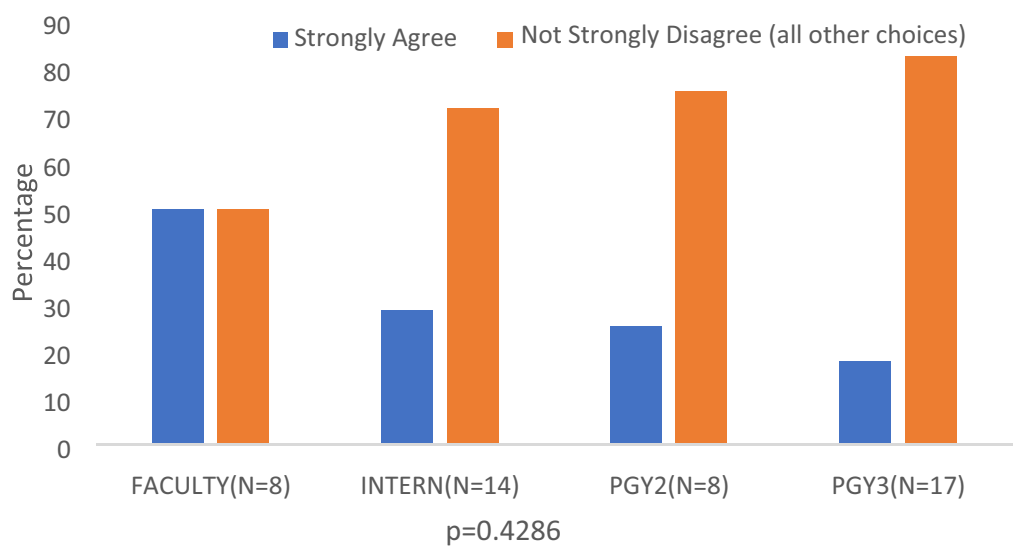


Figure 5. Provider responses to the statement "I felt comfortable managing patients via this modality." Abbreviation: PGY, postgraduate year.

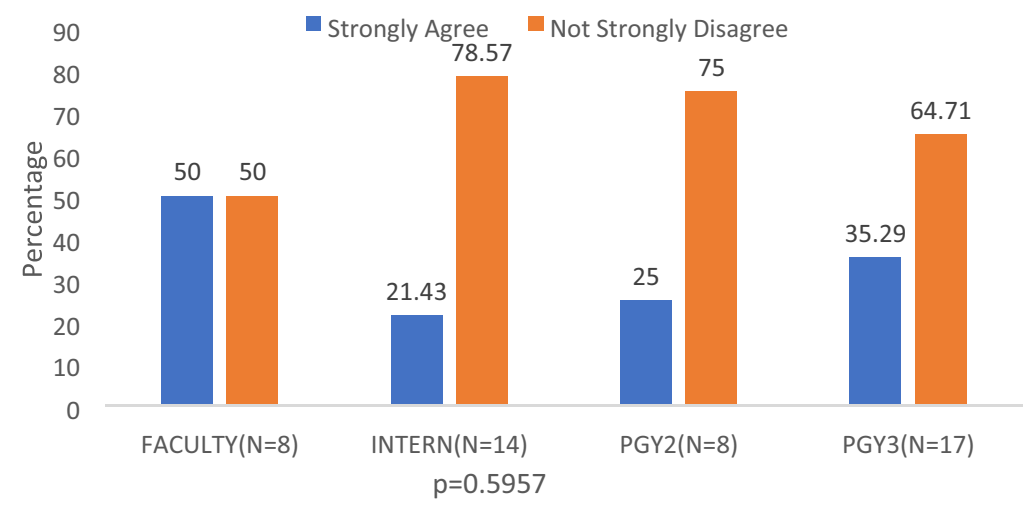

\section{Discussion}

Our results show that most patients who completed a virtual visit in the IM and FM clinics expressed a positive experience and would not mind continuing to have virtual visits in the future, even after the pandemic. This result correlates with many preceding studies that show a high satisfaction rate in patients using virtual visits. ${ }^{1}$ Similarly, positive experience was expressed by the majority of providers, although not as positively as for patients. This forced transition to telemedicine has had the effect of serving as a kind of stress test for hospital systems as to how the medical profession would benefit from continued use of audiovisual technology. The medical profession has successfully passed this stress test, and the result is that audiovisual technology is mutually beneficial to patients, providers, and hospital systems.
Previous studies have suggested that frontline health care providers may experience frustration with telehealth technology when the virtual modality does not meet their expectations. ${ }^{2}$ Although in health care physicians typically tend to be early adopters of change, who then educate their patient populations, in this case it appears that patients were more receptive of the change. There are several limitations to our study that need consideration. The first is that the IM providers exclusively used telephone, whereas the majority of FM providers used video visits. This would affect patient experience. Physical examination is the main limitation in both types of visits. In addition, the phone visits were limited by not having video so that patients and providers could see each other and note physical cues. In addition, the patient responses were collected by the providers themselves.

Figure 6. Provider survey responses to the statement "If I had a choice, I wouldn't mind seeing my patients in this way, at least a portion of the time." Abbreviation: PGY, postgraduate year.

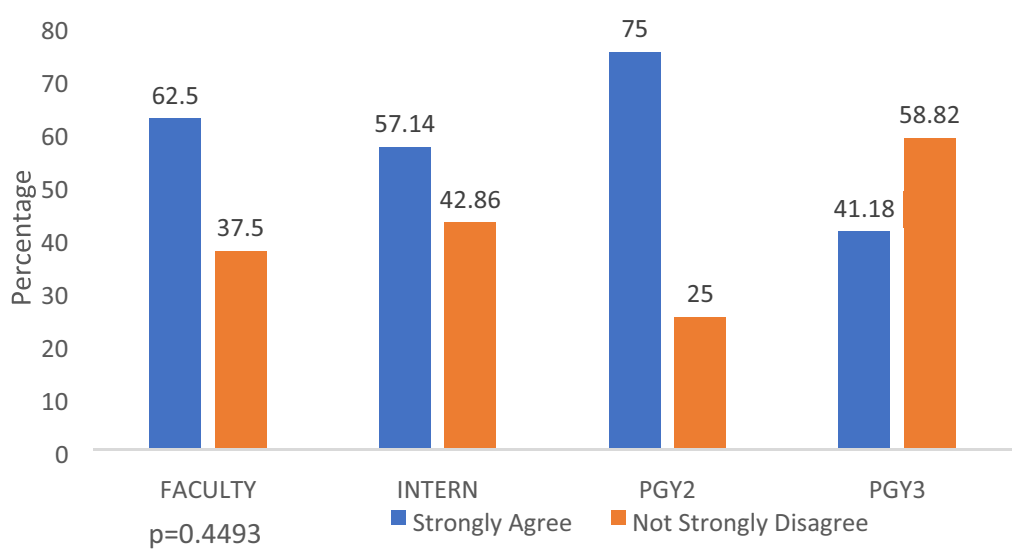


The patients could have felt compelled to give a favorable response. The patients also were the ones who agreed to a virtual visit in the first place, thus leading to a selection bias. They are more likely to rate it favorably and be willing to accept future virtual visits.

Older providers and interns had a less favorable response to virtual visits. The older providers were resistant to trying the new modality, whereas the interns were less confident in managing patients virtually. Surprisingly, the patients who rated their health as less than good preferred to come in for future visits and tended to not enjoy their virtual visits as much as the healthier patients. Rather than benefiting from not having to travel, especially during the pandemic, this could be due to the fact that these patients may perceive their health as needing more direct care due to their more complex health status.

\section{Conclusion}

The data tell us that the majority of patients (84.4\% for IM and 94\% for FM) enjoyed their virtual visits and would be open to continuing with virtual visits even after the COVID-19 pandemic (76.74\% of IM patients and $84.1 \%$ of FM patients). For providers, $82.89 \%$ of IM providers and $64 \%$ of FM providers agreed or strongly agreed that they enjoyed the visits, and $89.44 \%$ of IM providers and $88 \%$ of FM providers were open to continuing virtual visits after the pandemic. As to comfort, $91.4 \%$ of IM providers and $88 \%$ of FM providers felt comfortable managing visits virtually. Although the differences were not statistically significant, this shows patients are ahead of the curve in terms of adapting to telemedicine technology. Providers must be willing to adapt to meet patient demands.
Virtual visits were a necessary modality during the pandemic to continue outpatient primary care needs safely. Virtual visits allowed patients to continue care while keeping providers and staff safe and limiting use of personal protective equipment during a time when supplies were limited. As the pandemic continues, providers will continue to use audiovisual technology and work to improve the experience for both patients and providers. Studies have shown patients want more convenient access to providers with the use of audiovisual technology. Up until the COVID-19 pandemic, the majority of health systems have not met this request. Circumstances have shown it can continue to be a vital part of health care delivery. ${ }^{3}$

For assisting with biostatistics and creating tables, we thank Robert Mayberry, PhD, Department of Community Medicine and Preventive Health, Morehouse School of Medicine.

To see this article online, please go to: http://jabfm.org/content/ 34/Supplement/S71.full.

\section{References}

1. Pennic F. Survey: $83 \%$ of consumers are interested in receiving virtual care, but only $17 \%$ have access to it. HIT Consultant. May 22, 2019. Available from: https://hitconsultant.net/2019/05/22/survey-83-ofconsumers-are-interested-in-receiving-virtual-carebut-only-17-have-access-to-it/\#.XPGmsohKiUk.

2. MacNeill V, Sanders C, Fitzpatrick R, et al. Experiences of front-line health professionals in the delivery of telehealth: a qualitative study. Br J Gen Pract 2014;64:e401-e407.

3. Edwards L, Thomas C, Gregory A, et al. Are people with chronic diseases interested in using telehealth? A cross-sectional postal survey. J Med Internet Res 2014;16:e123. 